\title{
Microwave-assisted Protein Digestion on Various Locations of a Microplate
}

\author{
Ji-hye Lee ${ }^{a, b}$, Sehwan Park ${ }^{a}$, Sunyoung Lee ${ }^{a}$, Jangmi Hong ${ }^{a}$, Kyu Hwan Park ${ }^{b}$, Hyun Sik Kim ${ }^{b}$, and \\ Jeongkwon Kim ${ }^{\text {a,* }}$ \\ ${ }^{a}$ Department of Chemistry, Chungnam National University, Daejeon, South Korea \\ ${ }^{b}$ Korea Basic Science Institute, 804-1 Yangcheong-Ri, Ochang-Myun, Cheongwon-Gun, Chungcheongbuk-Do, 363-883, \\ Republic of Korea
}

Received December 9, 2011; Revised December 13, 2011; Accepted December 14, 2011

First published on the web December 15, 2011; http://dx.doi.org/10.5478/MSL.2011.2.4.084

\begin{abstract}
The effectiveness of microwave-assisted protein digestion in different well positions of a 96-well microplate was investigated where microwave-assisted protein digestion of bovine serum albumin was performed in 10 different wells of a 96-well microplate in a microwave oven. Similarly increased sequence coverages $(\sim 70 \%)$ were generally observed for the 10 microwaveassisted protein digestion samples compared to conventional overnight digestion (63\%), which is possibly due to higher miscleavage ratios $(\sim 53 \%)$ of the samples from microwave-assisted protein digestion than conventional overnight digestion (42.1\%). The reproducible results of microwave-assisted digestions from different well positions demonstrate the potential of high-throughput analysis of proteins using microwave-assisted protein digestion.
\end{abstract}

Keywords: Microwave, Mass spectrometry, MALDI, Microplate, Digestion

\section{Introduction}

In protein analysis, digestion of proteins using trypsin is an essential procedure to make small peptide fragments which are then analyzed by a mass spectrometer (MS), where overnight digestion at $37^{\circ} \mathrm{C}$ is a common digestion procedure. Recently, a lot of efforts have been exerted to expedite the digestion procedure, such as using microwave, ${ }^{1}$ ultrasound, ${ }^{2}$ or pressure. ${ }^{3}$ Previously our group has investigated the temperature effect on ultrasound-assisted digestion, ${ }^{4}$ the effect of vortex-induced vibrations on the digestion efficiency, ${ }^{5}$ and pressure-assisted digestion using a syringe. ${ }^{6}$ In a continuation of our study on protein digestion efficiency, the current study investigates the effectiveness of microwave-assisted protein digestion. Recently, microwave-assisted protein digestion ${ }^{7-9}$ is getting popular since microwave irradiation can significantly decrease digestion time, making the entire sample preparation fast and simple. Here we are investigating the effectiveness of microwaveassisted protein digestion in different well positions of a 96well microplate. A microwave oven was utilized to perform enzymatic digestion of a protein in 10 different well positions of a 96-well microplate. The digestion efficiencies of the samples in different well positions were compared by utilizing a matrix-assisted laser desorption/ionization time-of-flight (MALDI- TOF) MS.

*Reprint requests to Dr. Jeongkwon Kim

E-mail: jkkim48105@cnu.ac.kr

\section{Experimental}

\section{Materials}

Trifluoroacetic acid (TFA, $\geq 98 \%$ ), formic acid, $\alpha$-Cyano4-hydroxy-cinnamic acid, dithiothreitol (DTT, $\geq 99 \%$ ), iodoacetamide (IAA, 99\%), bovine serum albumin (BSA, $\geq 96 \%$ ), and acetonitrile (ACN) were purchased from SigmaAldrich (St. Louis, MO, USA). Sequence grade trypsin was obtained from Promega (Cat. No. v5113; Madison, WI, USA).

\section{Sample preparation}

Protein stock solutions were prepared by dissolving $10 \mathrm{mg}$ BSA in $1.0 \mathrm{~mL}$ of $50 \mathrm{mM}$ ammonium bicarbonate buffer solution. The denaturation of BSA was performed according to established procedures. ${ }^{6}$ Briefly, $10 \mathrm{mg}$ BSA was dissolved in $1 \mathrm{~mL}$ of $6 \mathrm{M}$ guanidine $\mathrm{HCl}$ in $50 \mathrm{mM}$ ammonium bicarbonate buffer ( $\mathrm{pH}$ 8.3). Then, $10 \mathrm{mM}$ DTT was added to the BSA solution. After reduction for $30 \mathrm{~min}$ at $56^{\circ} \mathrm{C}, 40 \mathrm{mM}$ IAA was added to the solution for alkylation in the dark for $30 \mathrm{~min}$ at room temperature, and subsequently the solution was desalted on a commercial YM-10 desalting spin column. The volume of the regained solution was adjusted to $1.0 \mathrm{~mL}$ using a micropipette. Then, $10 \mu \mathrm{L}$ of the BSA solutions $(10 \mathrm{mg} / \mathrm{mL})$ was mixed with $4.0 \mu \mathrm{L}$ of $0.5 \mu \mathrm{g} / \mu \mathrm{L}$ trypsin and $86 \mu \mathrm{L}$ of $50 \mathrm{mM}$ ammonium bicarbonate buffer (1:50 trypsin/protein ratio) in a 96-well plate. The final concentration of BSA sample solution was approximately $15 \mathrm{pmol} / \mu \mathrm{L}$.

To study the reproducibility of microwave-assisted digestion under different positions, tryptic digestion of BSA was performed in 10 random well positions (A1, A12, B9, C3, D6, E2, E10, 


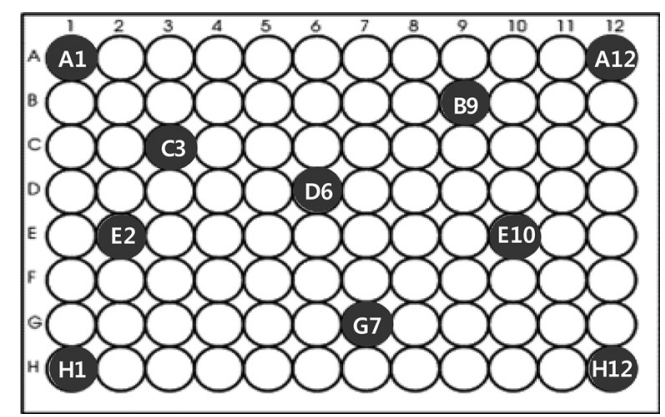

Figure 1. Sample locations in a 96-well microplate used for the current microwave-assisted digestion.

$\mathrm{G} 7, \mathrm{H} 1$, and $\mathrm{H} 12$ ) of a 96-well microplate for $10 \mathrm{~min}$ at $37^{\circ} \mathrm{C}$ with $400 \mathrm{~W}$ using a Microwave Digestion System (HST Rapid Enzyme Digestion System) from Hudson Surface Technology (Fort Lee, NJ, USA) as instructed in the user's manual. Figure 1 shows the 96-well microplate with sample locations used for this study. After the digestion, $1 \mu \mathrm{L}$ formic acid was added to each well to stop the reaction. For comparison, tryptic digestion of BSA was also performed using conventional overnight digestion at $37^{\circ} \mathrm{C}$.

\section{Mass spectrometry}

To prepare sample spots for MALDI-TOF MS analysis, $2 \mu \mathrm{L}$ of the sample solution was loaded onto a MALDI plate and dried. Then, $2 \mu \mathrm{L}$ of a CHCA matrix solution consisting of $10 \mathrm{mg}$ CHCA dissolved in $1 \mathrm{~mL}$ of water/ACN (50:50, v/v) containing $0.1 \%$ TFA was loaded onto each sample layer, and the digested protein was confirmed by MALDI-TOF MS. The mass spectrometer (Ultraflextreme time-of-flight mass spectrometer made by Bruker Daltonics, Germany) equipped with a 355-nm Nd:YaG laser was used to obtain mass spectra. Total shots were accumulated and mass spectra were obtained from $\mathrm{m} / \mathrm{z} 400-5000$ in a positive ion reflectron mode.

\section{Database searching}

The identification of digested peptides was done automated database searching of peak list files using the MASCOT search program (http://www.matrixscience.com). Database searches were restricted to the protein sequence of the corresponding protein downloaded from the SwissProt database. The following search parameters were selected for all database searching: enzyme, trypsin; missed cleavages, 5 ; peptide tolerance, \pm 0.5 $\mathrm{Da}$; mass values, $\mathrm{MH}^{+}$; fixed modifications, carbamidomethyl (C); variable modifications, oxidation (M). The search results, including unique peptide sequences, sequence coverage, calculated molecular mass of the peptide, were extracted.

\section{Results and Discussion}

\section{Digestion efficiency of microwave-assisted digestion in various locations}

In the current study, we are investigating the effectiveness of microwave-assisted protein digestion in different well positions of a 96-well microplate. Figure 1 shows the 10 random well positions where microwave-assisted digestion was performed (A1, A12, B9, C3, D6, E2, E10, G7, H1, and H12) on a 96-well microplate. Figure 2 displays the mass spectra of microwave-assisted digestion of BSA from the 10 well positions along with the mass spectrum of conventional overnight digestion, where similar patterns were observed for the former 10 mass spectra. In order to obtain detailed information, the peptide peaks were selected and searched using the MASCOT search program to identify the peaks. Supplementary table S1 exhibits the comprehensive information of the identified peptides from microwave-assisted digestion and conventional overnight digestion. Table 1 shows the summary of the comparison of digestion efficiency between microwave-assisted digestion at different well-positions and conventional overnight digestion.

As shown in Table 1, the sequence coverages of BSA from microwave-assisted digestion obtained from most well positions of the 96-well microplate were observed higher than that from conventional overnight digestion where the average sequence coverages from microwave-assisted digestions was $71.7 \%$, while the sequence coverage from overnight digestion was $63 \%$. The ratios of missed cleavages from the samples of the 10 well positions of the 96 -well microplate $(\sim 53.3 \%)$ were all higher than the ratio from overnight digestion $(42.1 \%)$. The increased sequence coverages from microwaveassisted digestion compared to the overnight digestion are believed to be due to the increased miscleavages. Miscleaved peptides contain additional basic amino acids, which assists the ionization of the peptides. ${ }^{10,11}$

\section{Comparison of miscleavage ratio between different digestion methods}

The average miscleavage ratio of the current ultrasoundassisted digestions was $53.3 \%$, while the miscleavage ratio of conventional overnight digestion was $42.1 \%$. The miscleavage ratios from so-called assisted digestions were always observed to be higher than those from conventional overnight digestion, such as pressure-assisted digestion using a syringe for $30 \mathrm{~min}$ at $37^{\circ} \mathrm{C}$ and $6 \mathrm{~atm}(43.2 \%$ vs. $24.2 \%),{ }^{6}$ vortex-assisted digestion for $1 \mathrm{hr}$ at $37^{\circ} \mathrm{C}$ with $2,500 \mathrm{rpm}(53.3 \%$ vs. $28.6 \%),{ }^{5}$ and ultrasound-assisted digestion at $55^{\circ} \mathrm{C}$ for $5 \mathrm{~min}(62.5 \%$ vs. $28.6 \%)^{4}$ for the analysis of BSA using MALDI-TOF MS. All of the above mentioned assist-digestion methods definitely expedite digestion process. However, the reduced digestion time is not long enough to reach a digestion status obtained from overnight digestion. Since the existence of miscleaved peptides generally provides more information of a protein by increasing the sequence coverage, the incomplete digestion may be more informative than complete digestion. The point is then to generate reproducible miscleaved peptides. The current analysis shows that the microwave-assisted digestion can provide reproducible digestion results. Reproducible digestion results can be useful for the quantitative analysis of proteins. 


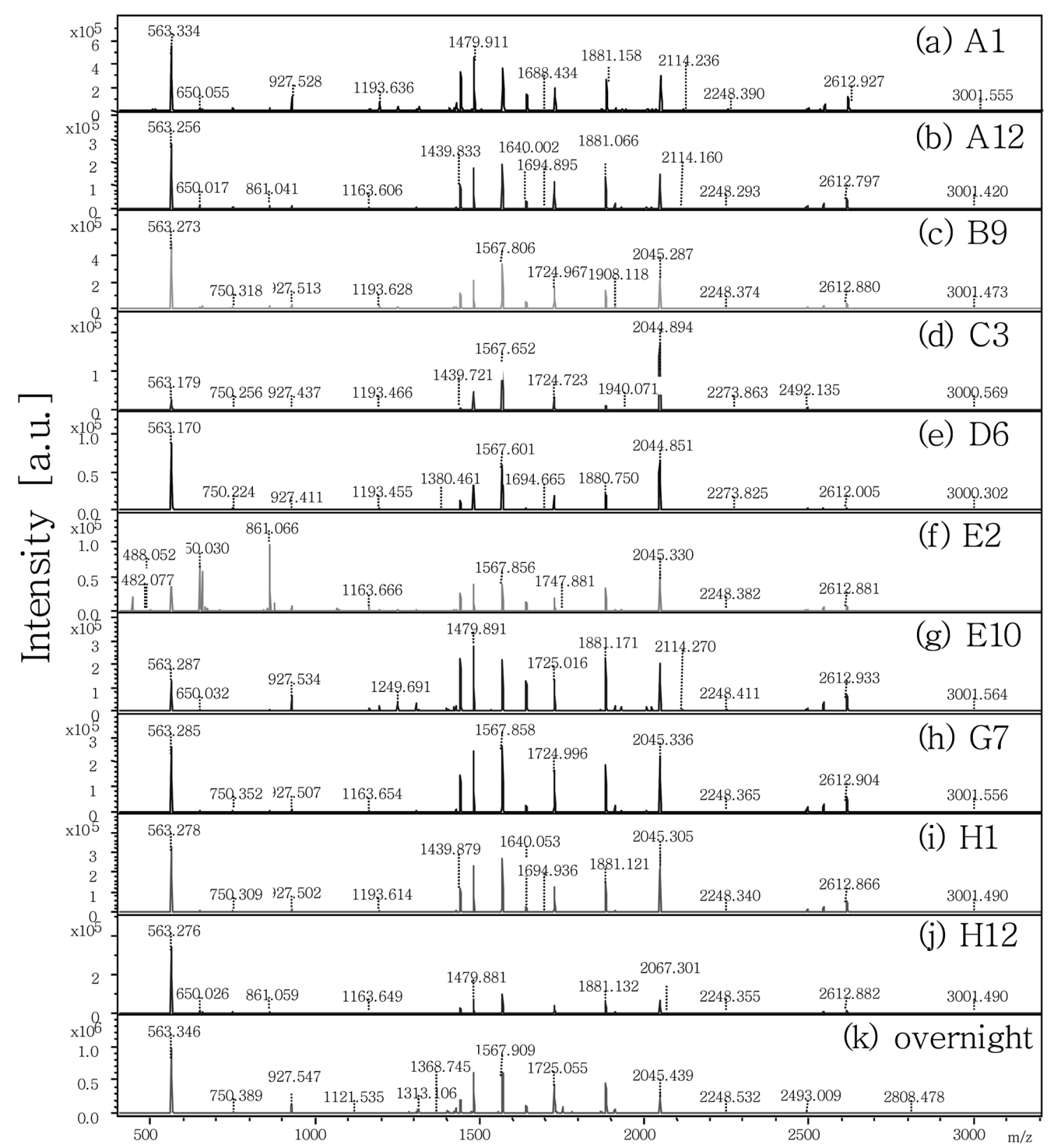

Figure 2. MALDI-TOF mass spectra of tryptically digested peptides of BSA produced by microwave-assisted digestion in 10 different well positions of a 96-well plate (a-j) and by conventional overnight digestion (k). Around 30 pmol was used for each mass spectrum. The exact sample locations are shown in Figure 1.

Table 1. Comparison of digestion efficiency between microwave-assisted digestion at different locations and conventional overnight digestion.

\begin{tabular}{ccccccccccccc}
\hline \hline & \multicolumn{1}{c}{ Microwave-assisted digestion at different locations } & \multicolumn{2}{c}{$\begin{array}{c}\text { Conventional } \\
\text { overnight digestion }\end{array}$} \\
\hline Position in a 96-well microplate & A1 & A12 & B9 & C3 & D6 & E2 & E10 & G7 & H1 & H12 & overn \\
\hline Sequence Coverage (\%) & 75 & 75 & 76 & 62 & 67 & 69 & 78 & 75 & 73 & 67 & 63 \\
Number of identified peptides & 55 & 55 & 57 & 35 & 36 & 47 & 60 & 56 & 55 & 43 & 38 \\
Number of miscleaved peptides & 29 & 29 & 31 & 21 & 20 & 24 & 31 & 30 & 29 & 21 & 16 \\
Ratio of missed cleavages (\%) & 52.7 & 52.7 & 54.4 & 60.0 & 55.6 & 51.1 & 51.7 & 53.6 & 52.7 & 48.8 & 42.1 \\
\hline
\end{tabular}

a) The ratio was calculated using the number of miscleaved peptides divided by the number of identified peptides. 
The small variation of the sequence coverage or the number of miscleaved peptides will be further reduced if some sort of shaking device is used during the microwave-assisted digestion process.

\section{Conclusion}

Microwave-assisted digestion for $10 \mathrm{~min}$ at $37^{\circ} \mathrm{C}$ with $400 \mathrm{~W}$ was performed for BSA in 10 different positions of a 96-well plate, where similar increased sequence coverage ( $\sim 70 \%)$ was observed for the 10 samples compared to conventional overnight digestion whose sequence coverage was $63 \%$. In terms of miscleavage, higher miscleavage ratios were observed from microwave-assisted digestion than conventional overnight digestion. The reproducible results from the 10 microwave-assisted digestions from different locations demonstrate that microwave can be successfully utilized for the quantitative analysis of protein samples.

\section{Supporting information}

Additional supporting information may be found in the online version of this article.

\section{Acknowledgments}

This research was supported by Basic Science Research Program through the National Research Foundation of Korea (NRF) funded by the Ministry of Education, Science and Technology (2010-0010776) to J.K. and by Korea Basic Science Institute (KBSI) grant G30122 to H.S.K.

\section{References}

1. Pramanik, B. N.; Mirza, U. A.; Ing, Y. H.; Liu, Y. H.; Bartner, P. L.; Weber, P. C.; Bose, A. K. Protein Sci. 2002, 11, 2676.

2. Lopez-Ferrer, D.; Capelo, J. L.; Vazquez, J. J. Proteome Res. 2005, 4, 1569.

3. Lopez-Ferrer, D.; Petritis, K.; Hixson, K. K.; Heibeck, T. H.; Moore, R. J.; Belov, M. E.; Camp, D. G., 2nd; Smith, R. D. J. Proteome Res. 2008, 7, 3276.

4. Shin, S.; Yang, H. J.; Kim, J.; Kim, J. Anal. Biochem. 2011, 414, 125.

5. Yang, H. J.; Shin, S.; Kim, J.; Hong, J.; Lee, S.; Kim, J. Rapid Commun. Mass Spectrom. 2011, 25, 88.

6. Yang, H. J.; Hong, J.; Lee, S.; Shin, S.; Kim, J.; Kim, J. Rapid Commun. Mass Spectrom. 2010, 24, 901.

7. Lin, S.; Yun, D.; Qi, D.; Deng, C.; Li, Y.; Zhang, X. J. Proteome Res. 2008, 7, 1297.

8. Sun, W.; Gao, S.; Wang, L.; Chen, Y.; Wu, S.; Wang, X.; Zheng, D.; Gao, Y. Mol. Cell. Proteomics 2006, 5, 769.

9. Ha, N. Y.; Kim, S. H.; Lee, T. G.; Han, S. Y. Langmuir 2011, 27, 10098.

10. Wu, S. L.; Kim, J.; Bandle, R. W.; Liotta, L.; Petricoin, E.; Karger, B. L. Mol. Cell. Proteomics 2006, 5, 1610.

11. Wu, S. L.; Kim, J.; Hancock, W. S.; Karger, B. J. Proteome Res. 2005, 4, 1155. 
Table S1. Peptide sequence information obtained from microwave-assisted tryptic digestion of BSA at $400 \mathrm{~W}$ for 10 min, along with conventional overnight digestion.

\begin{tabular}{|c|c|c|c|c|c|c|c|c|c|c|c|c|c|c|c|}
\hline \multirow{2}{*}{$\begin{array}{l}\text { Start- } \\
\text { End }\end{array}$} & \multirow[b]{2}{*}{ Peptide Sequence } & \multirow{2}{*}{$\begin{array}{l}\text { Number } \\
\text { of missed } \\
\text { cleavages }\end{array}$} & \multirow{2}{*}{$\operatorname{Mr}(\operatorname{expt})^{\mathrm{a})}$} & \multirow{2}{*}{$\operatorname{Mr}(\text { calc })^{b)}$} & \multicolumn{10}{|c|}{ Microwave-assisted digestion (400 W , $10 \mathrm{~min}$ ) } & \multirow{2}{*}{$\begin{array}{c}\text { Conventional } \\
\text { overnight } \\
\text { digestion }\end{array}$} \\
\hline & & & & & A1 & A12 & B9 & C3 & D6 & E2 & E10 & G7 & $\mathrm{H} 1$ & $\mathrm{H} 12$ & \\
\hline $20-24$ & R.GVFRR.D & 1 & 633.04 & 633.37 & & O & O & & & O & O & $\mathrm{O}$ & O & O & 0 \\
\hline $20-44$ & R.GVFRRDTHKSEIAHRFKDLGEEHFK.G & 5 & 3038.43 & 3038.56 & $\mathrm{O}$ & $\mathrm{O}$ & O & & & $\mathrm{O}$ & $\mathrm{O}$ & O & O & $\mathrm{O}$ & \\
\hline $24-28$ & R.RDTHK.S & 1 & 655.06 & 655.34 & $\mathrm{O}$ & $\mathrm{O}$ & $\mathrm{O}$ & $\mathrm{O}$ & $\mathrm{O}$ & $\mathrm{O}$ & $\mathrm{O}$ & $\mathrm{O}$ & $\mathrm{O}$ & $\mathrm{O}$ & \\
\hline $25-28$ & R.DTHK.S & 0 & 498.74 & 499.24 & $\mathrm{O}$ & O & $\mathrm{O}$ & & & O & $\mathrm{O}$ & $\mathrm{O}$ & O & $\mathrm{O}$ & \\
\hline $25-34$ & R.DTHKSEIAHR.F & 1 & 1192.67 & 1192.59 & $\mathrm{O}$ & O & $\mathrm{O}$ & $\mathrm{O}$ & $\mathrm{O}$ & O & O & $\mathrm{O}$ & O & $\mathrm{O}$ & $\mathrm{O}$ \\
\hline $35-44$ & R.FKDLGEEHFK.G & 1 & 1248.68 & 1248.61 & $\mathrm{O}$ & O & $\mathrm{O}$ & & $\mathrm{O}$ & O & O & $\mathrm{O}$ & O & $\mathrm{O}$ & $\mathrm{O}$ \\
\hline $45-65$ & K.GLVLIAFSQYLQQCPFDEHVK.L & 0 & 2491.74 & 2491.26 & $\mathrm{O}$ & $\mathrm{O}$ & $\mathrm{O}$ & $\mathrm{O}$ & $\mathrm{O}$ & $\mathrm{O}$ & $\mathrm{O}$ & $\mathrm{O}$ & $\mathrm{O}$ & $\mathrm{O}$ & \\
\hline $66-75$ & K.LVNELTEFAK.T & 0 & 1162.66 & 1162.62 & $\mathrm{O}$ & O & O & $\mathrm{O}$ & $\mathrm{O}$ & $\mathrm{O}$ & $\mathrm{O}$ & $\mathrm{O}$ & O & O & $\mathrm{O}$ \\
\hline $89-100$ & K.SLHTLFGDELCK.V & 0 & 1418.78 & 1418.69 & $\mathrm{O}$ & O & $\mathrm{O}$ & $\mathrm{O}$ & $\mathrm{O}$ & O & $\mathrm{O}$ & $\mathrm{O}$ & O & $\mathrm{O}$ & $\mathrm{O}$ \\
\hline $101-117$ & K.VASLRETYGDMADCCEK.Q & 1 & 2004.15 & 2003.84 & $\mathrm{O}$ & $\mathrm{O}$ & $\mathrm{O}$ & & $\mathrm{O}$ & $\mathrm{O}$ & $\mathrm{O}$ & $\mathrm{O}$ & $\mathrm{O}$ & $\mathrm{O}$ & \\
\hline $106-122$ & R.ETYGDMADCCEKQEPER.N & 1 & 2117.19 & 2116.81 & $\mathrm{O}$ & O & O & & & O & $\mathrm{O}$ & O & O & & \\
\hline 106-138 & R.ETYGDMADCCEKQEPERNECFLSHKDDSPDLPK.L & 3 & 4000.02 & 3999.67 & O & O & O & O & O & O & $\mathrm{O}$ & $\mathrm{O}$ & O & $\mathrm{O}$ & \\
\hline $118-130$ & K.QEPERNECFLSHK.D & 1 & 1672.94 & 1672.76 & O & O & $\mathrm{O}$ & & & O & $\mathrm{O}$ & $\mathrm{O}$ & & & $\mathrm{O}$ \\
\hline 118-138 & K.QEPERNECFLSHKDDSPDLPK.L & 2 & 2540.02 & 2540.16 & O & O & $\mathrm{O}$ & $\mathrm{O}$ & O & $\mathrm{O}$ & O & O & O & $\mathrm{O}$ & \\
\hline $123-138$ & R.NECFLSHKDDSPDLPK.L & 1 & 1901.15 & 1900.86 & $\mathrm{O}$ & $\mathrm{O}$ & $\mathrm{O}$ & & & $\mathrm{O}$ & $\mathrm{O}$ & $\mathrm{O}$ & $\mathrm{O}$ & & $\mathrm{O}$ \\
\hline $139-151$ & K.LKPDPNTLCDEFK.A & 0 & 1575.92 & 1575.76 & & & & & & & O & & & & $\mathrm{O}$ \\
\hline $139-155$ & K.LKPDPNTLCDEFKADEK.K & 1 & 2019.29 & 2018.96 & $\mathrm{O}$ & $\mathrm{O}$ & $\mathrm{O}$ & & O & $\mathrm{O}$ & $\mathrm{O}$ & $\mathrm{O}$ & $\mathrm{O}$ & $\mathrm{O}$ & \\
\hline $139-156$ & K.LKPDPNTLCDEFKADEKK.F & 2 & 2147.46 & 2147.06 & O & $\mathrm{O}$ & O & & O & O & O & $\mathrm{O}$ & O & O & \\
\hline $161-167$ & K.YLYEIAR.R & 0 & 926.53 & 926.49 & $\mathrm{O}$ & $\mathrm{O}$ & $\mathrm{O}$ & $\mathrm{O}$ & $\mathrm{O}$ & $\mathrm{O}$ & $\mathrm{O}$ & $\mathrm{O}$ & $\mathrm{O}$ & $\mathrm{O}$ & $\mathrm{O}$ \\
\hline $161-168$ & K.YLYEIARR.H & 1 & 1082.64 & 1082.59 & $\mathrm{O}$ & & $\mathrm{O}$ & & & O & O & $\mathrm{O}$ & O & & \\
\hline 168-183 & R.RHPYFYAPELLYYANK.Y & 1 & 2044.35 & 2044.02 & $\mathrm{O}$ & O & O & 0 & O & O & $\mathrm{O}$ & O & $\mathrm{O}$ & O & O \\
\hline 168-197 & R.RHPYFYAPELLYYANKYNGVFQECCQAEDK.G & 2 & 3772.95 & 3772.71 & & & & $\mathrm{O}$ & & & & & & & \\
\hline $169-183$ & R.HPYFYAPELLYYANK.Y & 0 & 1888.22 & 1887.92 & $\mathrm{O}$ & $\mathrm{O}$ & $\mathrm{O}$ & O & O & $\mathrm{O}$ & $\mathrm{O}$ & $\mathrm{O}$ & $\mathrm{O}$ & $\mathrm{O}$ & O \\
\hline 184-197 & K.YNGVFQECCQAEDK.G & 0 & 1746.92 & 1746.70 & $\mathrm{O}$ & $\mathrm{O}$ & O & & & $\mathrm{O}$ & O & $\mathrm{O}$ & O & O & \\
\hline
\end{tabular}




\begin{tabular}{|c|c|c|c|c|c|c|c|c|c|c|c|c|c|c|c|}
\hline 184-204 & K.YNGVFQECCQAEDKGACLLPK.I & 1 & 2485.95 & 2486.10 & & & & $\mathrm{O}$ & $\mathrm{O}$ & & & & & & \\
\hline 198-209 & K.GACLLPKIETMR.E Oxidation (M) & 1 & 1404.19 & 1403.73 & $\mathrm{O}$ & $\mathrm{O}$ & $\mathrm{O}$ & & & $\mathrm{O}$ & $\mathrm{O}$ & $\mathrm{O}$ & $\mathrm{O}$ & $\mathrm{O}$ & $\mathrm{O}$ \\
\hline $205-218$ & K.IETMREKVLASSAR.Q Oxidation (M) & 2 & 1606.08 & 1605.85 & & & $\mathrm{O}$ & & & & $\mathrm{O}$ & & & & \\
\hline 212-218 & K.VLASSAR.Q & 0 & 702.39 & 702.40 & $\mathrm{O}$ & $\mathrm{O}$ & $\mathrm{O}$ & & & $\mathrm{O}$ & $\mathrm{O}$ & $\mathrm{O}$ & O & $\mathrm{O}$ & $\mathrm{O}$ \\
\hline 219-222 & R.QRLR.C & 1 & 571.37 & 571.36 & & & & & & & & & & & $\mathrm{O}$ \\
\hline 223-228 & R.CASIQK.F & 0 & 705.35 & 705.35 & $\mathrm{O}$ & O & O & O & $\mathrm{O}$ & & O & $\mathrm{O}$ & $\mathrm{O}$ & O & $\mathrm{O}$ \\
\hline 229-232 & K.FGER.A & 0 & 507.29 & 507.24 & $\mathrm{O}$ & & $\mathrm{O}$ & & & & $\mathrm{O}$ & & & & \\
\hline 233-241 & R.ALKAWSVAR.L & 1 & 1000.64 & 1000.58 & $\mathrm{O}$ & O & $\mathrm{O}$ & & & & O & $\mathrm{O}$ & $\mathrm{O}$ & & \\
\hline 236-241 & K.AWSVAR.L & 0 & 688.41 & 688.37 & $\mathrm{O}$ & $\mathrm{O}$ & $\mathrm{O}$ & $\mathrm{O}$ & $\mathrm{O}$ & 0 & $\mathrm{O}$ & $\mathrm{O}$ & $\mathrm{O}$ & $\mathrm{O}$ & $\mathrm{O}$ \\
\hline 264-280 & K.VHKECCHGDLLECADDR.A & 1 & 2113.26 & 2112.88 & O & O & $\mathrm{O}$ & $\mathrm{O}$ & $\mathrm{O}$ & 0 & $\mathrm{O}$ & $\mathrm{O}$ & O & $\mathrm{O}$ & \\
\hline 264-285 & K.VHKECCHGDLLECADDRADLAK.Y & 2 & 2612.80 & 2612.16 & $\mathrm{O}$ & O & $\mathrm{O}$ & $\mathrm{O}$ & $\mathrm{O}$ & 0 & $\mathrm{O}$ & O & O & $\mathrm{O}$ & \\
\hline $267-280$ & K.ECCHGDLLECADDR.A & 0 & 1748.91 & 1748.66 & & & & & & & & & & & $\mathrm{O}$ \\
\hline 267-285 & K.ECCHGDLLECADDRADLAK.Y & 1 & 2247.40 & 2246.94 & O & O & $\mathrm{O}$ & & & 0 & $\mathrm{O}$ & O & O & $\mathrm{O}$ & \\
\hline 298-309 & K.LKECCDKPLLEK.S & 1 & 1531.92 & 1531.77 & O & O & $\mathrm{O}$ & & & $\mathrm{O}$ & $\mathrm{O}$ & O & $\mathrm{O}$ & & $\mathrm{O}$ \\
\hline 310-318 & K.SHCIAEVEK.D & 0 & 1071.09 & 1071.50 & $\mathrm{O}$ & $\mathrm{O}$ & $\mathrm{O}$ & & & $\mathrm{O}$ & $\mathrm{O}$ & $\mathrm{O}$ & $\mathrm{O}$ & $\mathrm{O}$ & $\mathrm{O}$ \\
\hline $310-340$ & K.SHCIAEVEKDAIPENLPPLTADFAEDKDVCK.N & 2 & 3510.87 & 3510.66 & & & & O & O & & & & & & \\
\hline $341-346$ & K.NYQEAK.D & 0 & 751.35 & 751.35 & $\mathrm{O}$ & $\mathrm{O}$ & $\mathrm{O}$ & $\mathrm{O}$ & $\mathrm{O}$ & O & $\mathrm{O}$ & $\mathrm{O}$ & O & $\mathrm{O}$ & $\mathrm{O}$ \\
\hline 347-359 & K.DAFLGSFLYEYSR.R & 0 & 1566.87 & 1566.74 & $\mathrm{O}$ & O & $\mathrm{O}$ & $\mathrm{O}$ & $\mathrm{O}$ & $\mathrm{O}$ & $\mathrm{O}$ & $\mathrm{O}$ & $\mathrm{O}$ & $\mathrm{O}$ & $\mathrm{O}$ \\
\hline $360-371$ & R.RHPEYAVSVLLR.L & 1 & 1438.91 & 1438.80 & $\mathrm{O}$ & $\mathrm{O}$ & $\mathrm{O}$ & O & O & 0 & O & O & $\mathrm{O}$ & $\mathrm{O}$ & $\mathrm{O}$ \\
\hline 361-371 & R.HPEYAVSVLLR.L & 0 & 1282.77 & 1282.70 & $\mathrm{O}$ & $\mathrm{O}$ & $\mathrm{O}$ & & & & $\mathrm{O}$ & $\mathrm{O}$ & $\mathrm{O}$ & & $\mathrm{O}$ \\
\hline 361-374 & R.HPEYAVSVLLRLAK.E & 1 & 1594.71 & 1594.92 & & & & $\mathrm{O}$ & & & & & & & \\
\hline 372-386 & R.LAKEYEATLEECCAK.D & 1 & 1814.05 & 1813.82 & O & $\mathrm{O}$ & $\mathrm{O}$ & & & & $\mathrm{O}$ & O & O & & \\
\hline $372-401$ & R.LAKEYEATLEECCAKDDPHACYSTVFDKLK.H & 3 & 3590.94 & 3590.64 & $\mathrm{O}$ & $\mathrm{O}$ & $\mathrm{O}$ & $\mathrm{O}$ & & & $\mathrm{O}$ & O & $\mathrm{O}$ & & \\
\hline $375-386$ & K.EYEATLEECCAK.D & 0 & 1501.79 & 1501.61 & O & $\mathrm{O}$ & O & O & $\mathrm{O}$ & & 0 & O & O & $\mathrm{O}$ & $\mathrm{O}$ \\
\hline $375-401$ & K.EYEATLEECCAKDDPHACYSTVFDKLK.H & 2 & 3278.45 & 3278.42 & & & & $\mathrm{O}$ & & & & & & & \\
\hline 387-399 & K.DDPHACYSTVFDK.L & 0 & 1553.82 & 1553.65 & O & $\mathrm{O}$ & $\mathrm{O}$ & & & $\mathrm{O}$ & O & O & O & O & 0 \\
\hline $387-401$ & K.DDPHACYSTVFDKLK.H & 1 & 1795.05 & 1794.82 & $\mathrm{O}$ & $\mathrm{O}$ & $\mathrm{O}$ & & & & $\mathrm{O}$ & $\mathrm{O}$ & $\mathrm{O}$ & $\mathrm{O}$ & $\mathrm{O}$ \\
\hline $402-412$ & K.HLVDEPQNLIK.Q & 0 & 1304.79 & 1304.71 & $\mathrm{O}$ & $\mathrm{O}$ & $\mathrm{O}$ & & $\mathrm{O}$ & $\mathrm{O}$ & $\mathrm{O}$ & $\mathrm{O}$ & $\mathrm{O}$ & $\mathrm{O}$ & $\mathrm{O}$ \\
\hline $413-420$ & K.QNCDQFEK.L & 0 & 1067.50 & 1067.43 & & & & & & & $\mathrm{O}$ & & & & \\
\hline
\end{tabular}




\begin{tabular}{|c|c|c|c|c|c|c|c|c|c|c|c|c|c|c|c|}
\hline $421-433$ & K.LGEYGFQNALIVR.Y & 0 & 1478.90 & 1478.79 & $\mathrm{O}$ & O & $\mathrm{O}$ & $\mathrm{O}$ & $\mathrm{O}$ & $\mathrm{O}$ & $\mathrm{O}$ & $\mathrm{O}$ & $\mathrm{O}$ & O & $\mathrm{O}$ \\
\hline 434-436 & R.YTR.K & 0 & 438.02 & 438.22 & & $\mathrm{O}$ & $\mathrm{O}$ & & & $\mathrm{O}$ & $\mathrm{O}$ & $\mathrm{O}$ & $\mathrm{O}$ & $\mathrm{O}$ & \\
\hline $437-451$ & R.KVPQVSTPTLVEVSR.S & 1 & 1639.09 & 1638.93 & $\mathrm{O}$ & $\mathrm{O}$ & O & $\mathrm{O}$ & $\mathrm{O}$ & $\mathrm{O}$ & $\mathrm{O}$ & $\mathrm{O}$ & $\mathrm{O}$ & O & $\mathrm{O}$ \\
\hline $460-468$ & R.CCTKPESER.M & 0 & 1165.55 & 1165.49 & $\mathrm{O}$ & O & $\mathrm{O}$ & & & $\mathrm{O}$ & $\mathrm{O}$ & $\mathrm{O}$ & $\mathrm{O}$ & & $\mathrm{O}$ \\
\hline $460-482$ & R.CCTKPESERMPCTEDYLSLILNR.L & 1 & 2871.45 & 2871.30 & & & & $\mathrm{O}$ & & & & & & & \\
\hline $469-482$ & R.MPCTEDYLSLILNR.L & 0 & 1724.01 & 1723.83 & $\mathrm{O}$ & O & $\mathrm{O}$ & & $\mathrm{O}$ & $\mathrm{O}$ & $\mathrm{O}$ & $\mathrm{O}$ & $\mathrm{O}$ & $\mathrm{O}$ & $\mathrm{O}$ \\
\hline 469-482 & R.MPCTEDYLSLILNR.L Oxidation (M) & 0 & 1740.05 & 1739.82 & $\mathrm{O}$ & $\mathrm{O}$ & & $\mathrm{O}$ & & $\mathrm{O}$ & $\mathrm{O}$ & $\mathrm{O}$ & $\mathrm{O}$ & & \\
\hline $483-507$ & R.LCVLHEKTPVSEKVTKCCTESLVNR.R & 3 & 2986.41 & 2986.50 & O & $\mathrm{O}$ & $\mathrm{O}$ & $\mathrm{O}$ & & & $\mathrm{O}$ & $\mathrm{O}$ & $\mathrm{O}$ & & \\
\hline 499-507 & K.CCTESLVNR.R & 0 & 1137.56 & 1137.49 & 0 & 0 & $\mathrm{O}$ & & & $\mathrm{O}$ & 0 & $\mathrm{O}$ & $\mathrm{O}$ & & \\
\hline 499-523 & K.CCTESLVNRRPCFSALTPDETYVPK.A & 2 & 3001.42 & 3000.40 & $\mathrm{O}$ & $\mathrm{O}$ & $\mathrm{O}$ & $\mathrm{O}$ & $\mathrm{O}$ & & $\mathrm{O}$ & $\mathrm{O}$ & $\mathrm{O}$ & $\mathrm{O}$ & $\mathrm{O}$ \\
\hline $508-523$ & R.RPCFSALTPDETYVPK.A & 0 & 1880.16 & 1879.91 & $\mathrm{O}$ & O & $\mathrm{O}$ & $\mathrm{O}$ & $\mathrm{O}$ & $\mathrm{O}$ & $\mathrm{O}$ & $\mathrm{O}$ & $\mathrm{O}$ & $\mathrm{O}$ & $\mathrm{O}$ \\
\hline $524-544$ & K.AFDEKLFTFHADICTLPDTEK.Q & 1 & 2497.31 & 2497.18 & & & & $\mathrm{O}$ & $\mathrm{O}$ & & & & & & \\
\hline $529-544$ & K.LFTFHADICTLPDTEK.Q & 0 & 1907.18 & 1906.91 & $\mathrm{O}$ & $\mathrm{O}$ & $\mathrm{O}$ & $\mathrm{O}$ & $\mathrm{O}$ & $\mathrm{O}$ & $\mathrm{O}$ & $\mathrm{O}$ & $\mathrm{O}$ & $\mathrm{O}$ & $\mathrm{O}$ \\
\hline $545-557$ & K.QIKKQTALVELLK.H & 2 & 1510.99 & 1510.94 & & & & & & & & & & & $\mathrm{O}$ \\
\hline $548-557$ & K.KQTALVELLK.H & 1 & 1141.43 & 1141.71 & $\mathrm{O}$ & $\mathrm{O}$ & $\mathrm{O}$ & $\mathrm{O}$ & $\mathrm{O}$ & $\mathrm{O}$ & $\mathrm{O}$ & $\mathrm{O}$ & $\mathrm{O}$ & $\mathrm{O}$ & $\mathrm{O}$ \\
\hline $569-580$ & K.TVMENFVAFVDK.C & 0 & 1398.77 & 1398.69 & $\mathrm{O}$ & $\mathrm{O}$ & $\mathrm{O}$ & & $\mathrm{O}$ & $\mathrm{O}$ & $\mathrm{O}$ & $\mathrm{O}$ & $\mathrm{O}$ & O & $\mathrm{O}$ \\
\hline $569-597$ & K.TVMENFVAFVDKCCAADDKEACFAVEGPK.L & 2 & 3307.52 & 3307.47 & & & & $\mathrm{O}$ & $\mathrm{O}$ & & & & & & \\
\hline $581-597$ & K.CCAADDKEACFAVEGPK.L & 1 & 1927.07 & 1926.79 & $\mathrm{O}$ & $\mathrm{O}$ & $\mathrm{O}$ & & $\mathrm{O}$ & $\mathrm{O}$ & $\mathrm{O}$ & $\mathrm{O}$ & $\mathrm{O}$ & O & $\mathrm{O}$ \\
\hline \multicolumn{5}{|c|}{ Sequence Coverage (\%) } & 75 & 75 & 76 & 62 & 67 & 69 & 78 & 75 & 73 & 67 & 63 \\
\hline
\end{tabular}

a) Experimental $\mathrm{m} / \mathrm{z}$ transformed to a relative molecular mass

b) Relative molecular mass calculated from the matched peptide sequence 